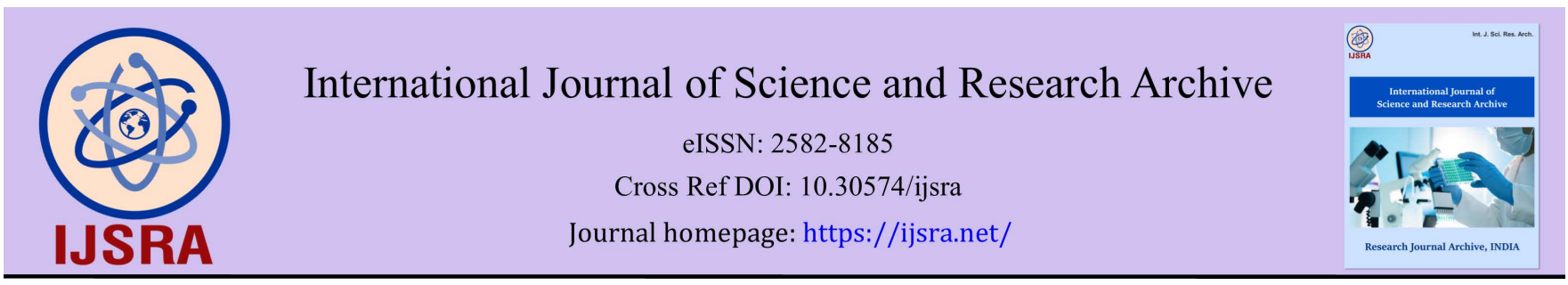

(CASE REPORT)

Check for updates

\title{
Kimura's disease: an extremely rare case report in an elderly male, followed by Hodgkin's disease
}

\author{
Nupur Rastogi * \\ M.D. Pathology, Sr. Consultant Pathologist, Private Diagnostic Centre, Kota, Rajasthan, India
}

International Journal of Science and Research Archive, 2021, 02(01), 156-159

Publication history: Received on 15 January 2021; revised on 20 February 2021; accepted on 22 February 2021

Article DOI: https://doi.org/10.30574/ijsra.2021.2.1.0030

\begin{abstract}
Kimura disease is a benign rare chronic inflammatory disorder of unknown etiology. The disease is mainly endemic in Asia, cases have been reported in Europe and America. The present case is of a 75 year Indian male who presented with cervical and axillary lymphadenopathy. Ultrasonography was done which showed multiple peritoneal lymph nodes. Peripheral smear showed eosinophilia. Cytomorphology and histopathology showed features of Hodgkin's disease. S IgE level was raised.
\end{abstract}

5 years earlier patient had presented with similar complaints of generalized lymphadenopathy, raised IgE level, peripheral eosinophilia. FNAC of cervical lymph node done earlier showed cytomorphology of Reactive Lymphadenitis. Biopsy showed histology of Kimura's disease.

Keywords: Kimura's disease; Hodgkin's lymphoma; Peripheral eosinophilia; Raised IgE levels; Generalized lymphadenopathy

\section{Introduction}

Kimura's disease is a benign chronic inflammatory disorder attributed to an immune- mediated hypersensitivity [1]. Kimura's disease was first reported by Kimm and Szeto in 1937 in China, but the disease was coined as Kimura's disease when in 1948, a Japanese doctor named Kimura et al published a systemic description of the disease [2,3]. Kimura's disease typically involves deep subcutaneous tissue and lymph nodes of the head and neck region with frequent regional lymphadenopathy and occasional salivary gland enlargement [4]. KD is rare in India, nearly 200 cases have been reported worldwide [5]. It is seen in young adults mostly second to fourth decade, male female ratio being 3:1 [6]. Common sites of involvement are periauricular region, groin, orbit and eyelids. Peripheral blood eosinophilia and increased serum immunoglobulin E (IgE) levels are always seen in Kimura's disease [7]. It has to be differentiated from other conditions mainly lymphoma. We report a case of 72 years male who had unusual presentation of generalised lymphadenopathy.

\section{Case report}

A 72 year old male patient presented with cervical and axillary lymph nodes enlargement, mild fever, itching. Fine needle aspiration cytology from axillary lymph node was done. Cytosmears showed lymphocytes, large binucleate, multinucleate cells with prominent eosinophilic nucleolus (resembling R-S cells), few eosinophils (fig 1). S IgE level was done which was raised. Also there was peripheral eosinophilia of about $18 \%$. Ultrasonography of abdomen showed multiple intraabdominal lymph nodes. Biopsy was done from axillary lymph node, the histological sections showed

\footnotetext{
${ }^{*}$ Corresponding author: Dr. Nupur Rastogi

M.D. Pathology, Sr. Consultant Pathologist, Private Diagnostic Centre, Kota, Rajasthan, India.
}

Copyright (C) 2021 Author(s) retain the copyright of this article. This article is published under the terms of the Creative Commons Attribution Liscense 4.0. 
effacement of lymph node architecture with presence of R-S cells , popcorn cells , lymphocytes, eosinophils and few plasma cells . Further Immunohistochemistry was done which was positive for CD 15, CD 30, PAX5 weak positive, Negative for CD20, CD3, CD10, CD23, CD5, thus confirming the diagnosis of Classical Hodgkin's lymphoma-mixed cellularity type. PET-CT scan showed left supraclavicular lymph node, multiple enlarged left axillary, deep pectoral nodes, multiple portocaval, paraduodenal, pancreatic, pre aortic, aorto caval nodes.

On taking the detail history, patient had presented with generalized lymphadenopathy and itching, fever 5 years earlier. Biopsy from cervical lymph node done 5 years back elsewhere was studied retrospectively. Cytological diagnosis of Reactive lymphadenitis was given at that time (Fig 2). H\&E sections of previous biopsy showed follicular hyperplasia with interfollicular increase in eosinophils and occasional eosinophilic abscess (Fig 3). Old records showed peripheral eosinophilia of $22 \%$ and increase in S. IgE levels. Patient was treated with steroids for 21 days. Previously diagnosis was not established. On retrospective study of the previous H\&E section of biopsy and related clinical and other laboratory findings, diagnosis of Kimura's disease was made.

This is a very rare presentation of Kimura's disease in an elderly patient presenting with generalized lymphadenopathy, peripheral eosinophilia and increase in S. IgE level. With similar presentation of generalised lymphadenopathy after 5 years with development of Hodgkin's disease also presenting with peripheral eosinophilia and increase S. IgE levels. Presently the patient has completed chemotherapy for Hodgkin's disease with complete remission of the lymph nodes.

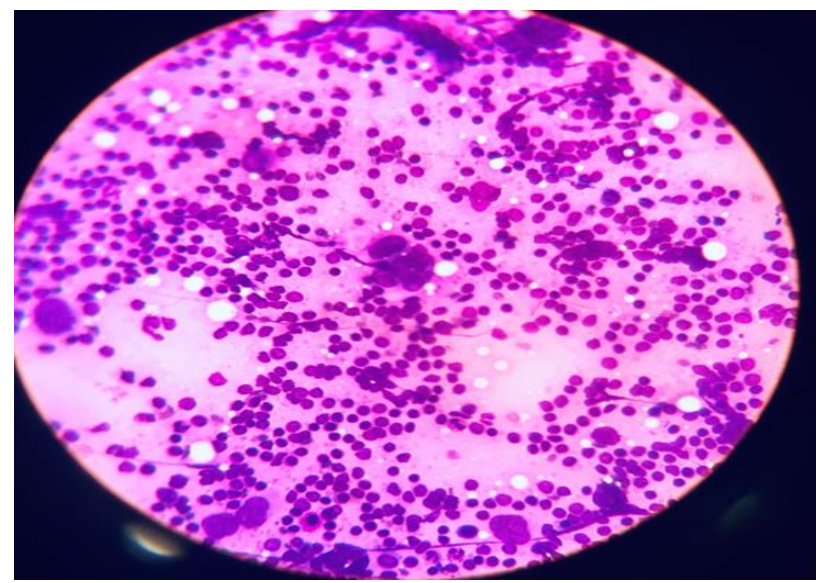

Figure 1 Giemsa stain, 10 X. Cytology smears taken 5 years later from axillary lymph node showing R-S cells and cytomorphology of Hodgkin's lymphoma-mixed cellularity

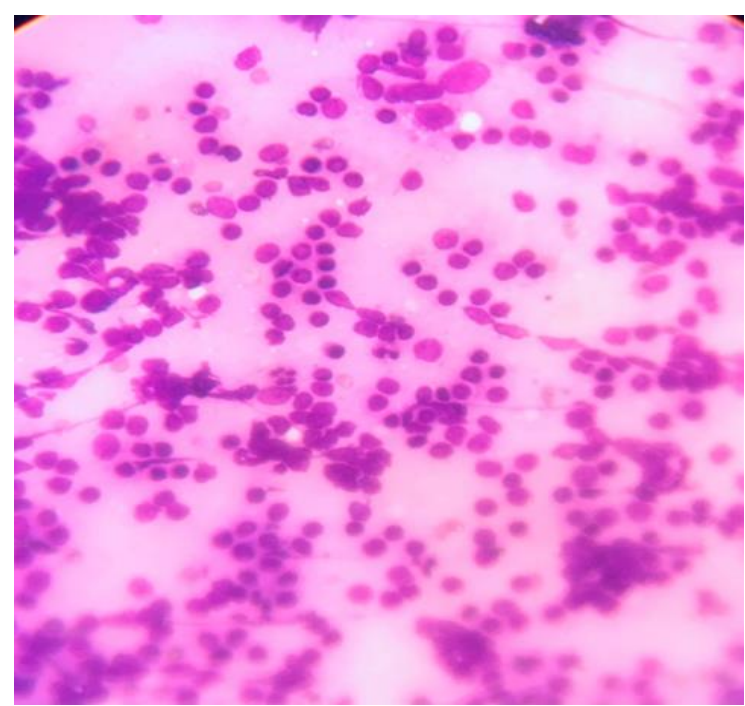

Figure 2 Cytology showing eosinophils 


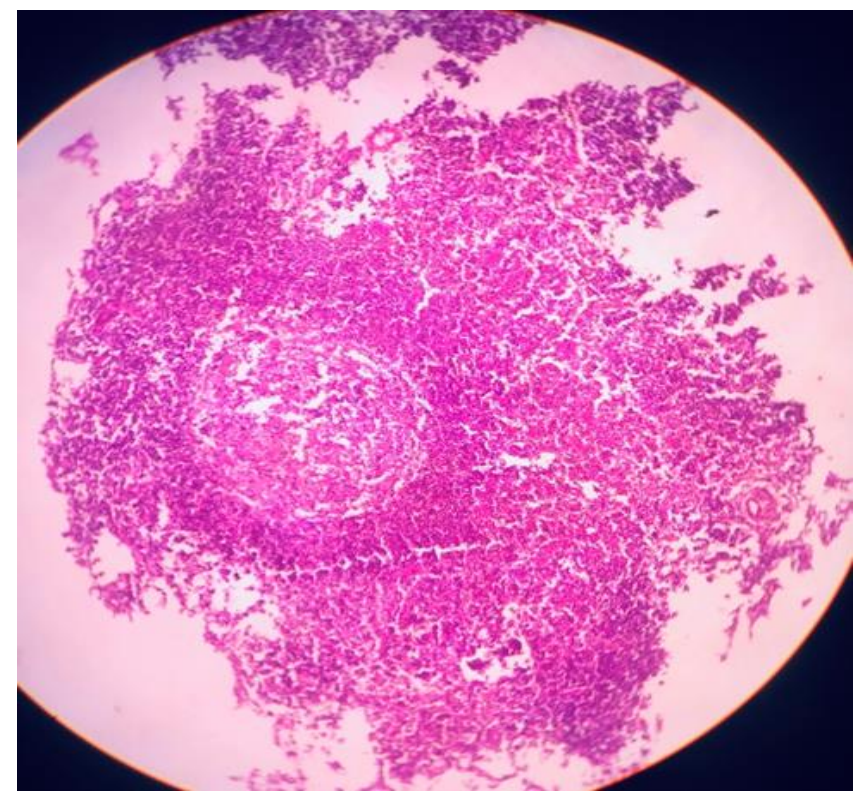

Figure 3 H\& E stain 10 X, Kimura disease

\section{Discussion}

Kimura disease was first described in 1937 by Kim and Szeto (China) as eosinophilic hyperplastic granuloma, its histological description was given by Kimura (Japan) in 1948[2,3]. Kimura disease is endemic in parts of Asia (Japan, China and Indonesia), although few cases have been reported in Europe and America. Kimura disease is rare in India with about 200 cases been reported worldwide [5]. Male female ratio being 3:1, more commonly seen in second to fourth decade. It presents predominantly as subcutaneous nodules in the head and neck, often unilateral, sometimes bilateral, frequently associated with regional lymphadenopathy with/without the involvement of salivary glands. Orbit, eyelid, palate, pharynx may be involved alongwith axilla, groin, arm [7,8]. The clinical course is benign and self limited, allergic or due to autoimmune response triggered by unknown persistent stimulus with occasional spontaneous resolution. There is proliferation of CD4 T-helper cells with overproduction of granulocyte macrophage colonystimulating factor, tumor necrosis factor-alpha, IL-4, IL-5, eotaxin which trigger the production of lymphoid follicle and high IgE [9]. There is no specific diagnostic feature of Kimura disease. FNAC is helpful in pre operative diagnosis of Kimura disease.

Hui et al classified three histological features of K.D. as constant, frequent and rare [8,10]. Constant features include preseverd nodal architecture, florid germinal centre hyperplasia, eosinophilic infiltration and post capillary venule proliferation. Frequent features comprise sclerosis, polykaryocytes, vascularization of the germinal centres, necrosis of the germinal centres, eosinophilic abscesses and reticular IgE deposition within germinal centres. Solitary rare feature is the progressive transformation of the germinal centres. Nodal architecture is preserved but capsular fibrosis with subcapsular sinusoid obliteration and perinodal soft tissue involvement is frequently present [8]. KD has to be differentiated from angiolymphoid hyperplasia with eosinophilia (ALHE), Hodgkin's disease, Kaposi sarcoma, eosinophilic granuloma, epithelioid hemangioma, Castleman's disease, tuberculosis, dermatopathic lymphadenopathy, lymphadenopathy of drug reactions, parasitic lymphadenitis, eosinophilic granuloma, epithelioid hemangioma. KD is differentiated from ALHE by peripheral eosinophilia and raised S. IgE levels.

In contrast to $\mathrm{KD}$, vascular proliferation is most significant in ALHE, forming aggregates or lobules comprising of plump endothelial cells with epithelioid or histiocytoid changes demonstrating cytologic atypia and vacuolization [8\}. Histopathology is diagnostic for KD. Thus cytological features of reactive hyperplasia with the presence of eosinophils should clinch the diagnosis of KD at cytology.

In the present case as there was generalised lymphadenopathy, complete excision of all lymph nodes was not possible. Steroid therapy was given for which the patient responded completely.

Later after 5 years patient presented with similar complaints but was diagnosed as Hodgkin's disease. 


\section{Conclusion}

The present case of Kimura disease presenting with generalized lymphadenopathy, peripheral eosinophilia, raised S IgE, followed 5 years later with similar presentation of generalized lymphadenopathy and raised IgE, peripheral eosinophilia and development of Classical Hodgkin;s diserase - mixed cellularity type is very unusual. The author has not come across any literature with this kind of presentation and development of Hodgkin's lymphoma later. This could be a coincidental presentation.

\section{Compliance with ethical standards}

\section{Acknowledgments}

Patient and her relatives.

\section{Statement of informed consent}

Informed consent was obtained from all individual participants included in the study.

\section{References}

[1] Manish S, Formosan Journal of Surgery. 2018; 51(1): 26-28.

[2] Kim HT, Szeto C. Eosinophilic hyperplastic lymphogranuloma, comparison with Mikulicz's disease. Proc Chin Med Soc. $1937 ; 1: 329$.

[3] Kimura T, Yoshimura S, Ishikawa E. On the unusual granulation combined with hyperplastic changes of lymphatic tissue. Trans Soc Pathol Jpn. 1948; 37: 179-80.

[4] Meningaud JP, et al. Kimura's disease of the parotid region: Report of 2 cases and review of the literature. J Oral Maxillofac Surg. 2007; 65: 134-40.

[5] Rajesh A et al. Kimura's disease: A case presentation of postauricular swelling. Niger J Clin Pract. 2016; 19: 827830.

[6] Fouda MA et al. Kimura disease: a case report and review of the literature with a new management protocol. Int J Nephrol. 2011.

[7] Shetty AK et al. Kimura's disease: a diagnostic challenge. Pediatrics. 2002; 110.

[8] Chen H et al. Kimura disease: a clinicopathologic study of 21 cases. Am J Surg Pathol. 2004; 28: 505-513.

[9] Meningaud JP et al. Kimura's disease of the parotid region: report of 2 cases and review of the literature. J Oral Maxillofac Surg. 2007; 65: 134-140.

[10] Hui PK et al. Lymphadenopathy of Kimura's disease. Am J Surg Pathol. 1989; 13: 177-186. 